\title{
Progress in Modeling Pu Properties and Aging-ESC Review, March 19-23, 2001
}

W. G. Wolfer, M. Caturla, A. Kubota, A. Quong, B. Sadigh, $P$. Sterne, M. Surh, C. Schaldach, B. Wirth

\section{March 29, 2001}




\section{DISCLAIMER}

This document was prepared as an account of work sponsored by an agency of the United States Government. Neither the United States Government nor the University of California nor any of their employees, makes any warranty, express or implied, or assumes any legal liability or responsibility for the accuracy, completeness, or usefulness of any information, apparatus, product, or process disclosed, or represents that its use would not infringe privately owned rights. Reference herein to any specific commercial product, process, or service by trade name, trademark, manufacturer, or otherwise, does not necessarily constitute or imply its endorsement, recommendation, or favoring by the United States Government or the University of California. The views and opinions of authors expressed herein do not necessarily state or reflect those of the United States Government or the University of California, and shall not be used for advertising or product endorsement purposes.

This work was performed under the auspices of the U. S. Department of Energy by the University of California, Lawrence Livermore National Laboratory under Contract No. W-7405-Eng-48.

This report has been reproduced directly from the best available copy.

Available electronically at http://www.doc.gov/bridge

Available for a processing fee to U.S. Department of Energy And its contractors in paper from

U.S. Department of Energy

Office of Scientific and Technical Information

P.O. Box 62

Oak Ridge, TN 37831-0062

Telephone: (865) 576-8401

Facsimile: (865) 576-5728

E-mail: reports@adonis.osti.gov

Available for the sale to the public from

U.S. Department of Commerce

National Technical Information Service

5285 Port Royal Road

Springfield, VA 22161

Telephone: (800) 553-6847

Facsimile: (703) 605-6900

E-mail: orders@ntis.fedworld.gov

Online ordering: http://www.ntis.gov/ordering.htm

OR

Lawrence Livermore National Laboratory

Technical Information Department's Digital Library

http://www.llnl.gov/tid/Library.html 


\title{
Progress in Modeling Pu Properties and Aging
}

\author{
W. G. Wolfer, Maria Caturla, Allison Kubota, Andrew Quong, \\ Babak Sadigh, Phil Sterne, Mike Surh, Charlene Schaldach, \& \\ Brian Wirth
}

\author{
ESC Review , March 19-23, 2001, Livermore \\ Numbers in [ ] refer to attached viewgraphs
}

[1] An important component of the Pu aging programs both here at LLNL and at LANL is the development of models for the aging processes. These models will, in the foreseeable future, be validated with more and more data from different experiments, and eventually enable us to predict the lifetime of pits.

The processes of aging from within the material are mainly caused by the radioactive decay of $\mathrm{Pu}$, and this produces three drivers for changes in properties :

1) Radiation damage in the form of displacements, of defects, of dislocations, and voids.

2) Helium will aggregate in the form of bubbles and cavities.

3) Uranium, Americium, and Neptunium will change the chemical composition and perhaps the phase stability.

All the three drivers act synergistically, no doubt. But it is useful to approach the complexity of Pu aging with the strategy of "first divide, conquer, and then unify".

[2] In this spirit, we have divided the program into three major task areas with individual subtasks to be conquered by various researchers.

The subtasks and staffing has changed somewhat from last year, and this is indicated on the viewgraph by underlines. Undoubtedly, it will change in the future, but the objectives of the major tasks and of the entire project will not change until the mission as stated at the beginning will have been accomplished. As you see from the third task, there is a strong emphasis on providing theoretical and computational support for critical, fundamental experiments.

Before reviewing the progress made last year for the various tasks, let me briefly state what the physical properties are that we monitor for signs of aging. Prominent is the change in density and microstructure, and its effects on strength and EOS. You will hear little if anything at all about the latter two 
properties, but work is in progress on these issues, and the review committee will hear about it at the next review. For this review, I want to concentrate on dimensional changes and the underlying changes in microstructure. Once we understand the changes in the microstructure, we can perhaps predict its influence on strength and EOS. It forms the basis for the predictive capability that we want to develop.

[3] Our present view of density changes is that there are four major contributions :

two arise from the displacement damage, one from helium bubbles, and the fourth from phase changes, mainly the martensitic conversion of delta to alpha. The radiation-induced changes are twofold, a change in lattice parameter, and a change due to void swelling. Of these four, only two have been confirmed experimentally so far. No evidence exists yet for void swelling or phase changes.

[4] The initial process of radiation damage is the collision cascade. Because of the large recoil energy of the $U$ atom, $85 \mathrm{keV}$, the cascade energizes about 20,000 atoms. Therefore, MD simulations in a very large system need to be carried out. We had performed such simulations on 10 million $\mathrm{Pb}$ atoms in the past year.

[5] This year, we spent our efforts on implementing a Pu potential in the radiation damage codes. We used the MEAM potential of Mike Baskes. Because it has an angular dependence, it is computationally much more demanding. We have accomplished to incorporate this potential and achieve excellent scaling on the ASCI parallel machine. Some simulations on small systems have already been performed. For example, we have computed displacement energies for $\mathrm{Pu}$, and they are somewhat smaller on average than the value of 14 eV used in our earlier assessments.

[6] The defect clusters that are retained after the cascade cools off, are, as we have conjectured, responsible for the transient lattice parameter change. The accumulation of the defect clusters generated in cascades will have to reach a saturation density. Two processes limit the build-up of this density: the rate of thermal annealing or dissolution of clusters becomes eventually comparable to the rate of generation, and/or newly generated cascades obliterate the defect clusters from earlier cascades.

[7] The latter process was not included in our earlier simulations but has now been incorporated during the past year. It turns out to be important. 


\section{UNCLASSIFIED}

The results shown are for copper where TEM data exist on the defect cluster accumulation. Our new results clearly show the transition to saturation. Making the connection between cluster density and lattice parameter change is, however, difficult since only one experimental result from unpublished work by Steve Zinkle (ORNL) is available. This result indicates that the density of clusters visible in TEM may be significantly lower than the total cluster density that contributes to the lattice parameter change. The resolution of this discrepancy will be the subject for further analysis.

There is an even larger discrepancy between the cluster density computed for Pu and the reported lattice parameter changes from Russian work. Part of the problem is that we do not know the pressure derivative of the elastic constants for d-Pu which determine the lattice parameter change produced by defect clusters. We think that we can compute these pressure derivatives from first principles with our electronic structure methods developed during the past years. These methods are also needed for other reasons.

There are many parameters for the defects produced in Pu which will most likely never be measured directly. It would be too costly or simply not possible with Pu. As a result, we have from the very beginning of this project aimed at developing first principle methods to explain the physical origin for the many phases of $\mathrm{Pu}$, and in particular to explain the existence of the delta phase with its low density. The first principle methods will also enable us to get values for defect parameters not accessible to direct experimental determination.

A recent breakthrough was made by Per Soderlind from the Physics Directorate here at LLNL. He showed that it is necessary to include spin-polarization in the electronic structure calculation for $\mathrm{Pu}$, just as it is required for the transition metals and the lanthanides. We have adopted this approach, but in addition, developed pseudopotentials for Pu to avoid having to do full-potential calculations with all 94 electrons included explicitly in the calculations. We have made significant progress in achieving this goal.

[8] The pseudo-potential for the 6s, 6p, and $5 f$ electrons are shown for Pu. Notice that the $5 f$ electrons still see a deep potential well deep within the atomic core of $\mathrm{Pu}$, and as a result, they can be strongly localized as they indeed are in d-Pu.

[9] The spin-polarized theory gives now very satisfactory results for both the lattice parameter and the bulk modulus of the d-phase. Without spinpolarization, the atomic volumes and the bulk modulii of a-Pu and d-Pu are predicted to be very nearly the same. In reality, they differ by $20 \%$. 
To provide experimental information on the electronic structure in the alpha and delta phases of $\mathrm{Pu}$, photo-electron emission experiments are carried out by Jim Tobin and collaborators at LANL and LBL. Their work is presented in a poster.

The most significant accomplishment during the last year was the experimental discovery of nanometer helium bubbles in aged Pu. Adam Schwartz will present this exciting discovery tomorrow. Although helium-vacancy clusters and small bubbles were conjectured earlier, there was no direct evidence. We have clear evidence now.

[10] As a result of this discovery, we have begun in earnest to model helium bubble formation.

[11] I present a simple model intended to scope the problem, and show how surprisingly successful this model turns out to be, at least so far.

[12] One of the most surprising results is that the majority of the bubbles nucleate within one year, and they then become the scavengers for all the helium born thereafter. Although the bubbles grow with age, their diameter increases only with the cubic root of the total helium concentration. The helium concentration in solution, $i$. e. not in bubbles, goes though a sharp maximum at the tender age of one day, and then drops precipitously to concentrations of ppt. This drop coincides with the nucleation burst of helium bubbles.

[13] Temperature and 238 enrichment affect the density of helium bubbles. Fortunately, and somewhat by luck, we had selected an accelerated aging experiment at $65 \mathrm{C}$, which is equivalent to natural aging at $35 \mathrm{C}$ with regard to helium bubble formation. Note, however, that the equivalent accelerated aging temperature for void swelling is $50 \mathrm{C}$. Our accelerated aging experiments will be conducted at the three temperatures of $35 \mathrm{C}, 50 \mathrm{C}$, and $65 \mathrm{C}$.

[14] Because these bubbles are so small, the pressure of the 200 to 300 helium atom fluid is high, in the GPa range. But given the equally large surface tension, they are in fact close to being equilibrium bubbles.

[15] This has important implications. One is that heating the material to $2 / 3$ of the melting point or below will not drastically change the bubble size or lower the helium density dramatically. This has been confirmed by Rich Howell with 
PAS studies, and I urge you to view his poster for details of this interesting experiment as well as other experiments.

[16] There is another interesting piece of information that can be extracted from this model. The helium atom is assumed to reside in a vacancy and to diffuse with the vacancy. The activation energy is, to a first approximation, assumed to be the same as for the empty vacancy. With a vacancy migration energy of $0.68 \mathrm{eV}$, the model generates a bubble density in agreement with the TEM observations.

With an activation energy of $0.54 \mathrm{eV}$, corresponding to Stage III in the isochronal annealing experiments of Fluss et al., however, the bubble density comes out to be too low by an order of magnitude. Mike Fluss will present tomorrow the results and analysis of the isochronal annealing experiments for damage produced at liquid helium temperatures and generated by either selfirradiation or proton bombardment.

[17] Finally, the helium bubble model implies a small volume expansion roughly proportional to the total amount of helium generated. The expansion is on the order of one atomic volume of Pu per helium atom. Immersion density measurements on old stockpile material are consistent with this prediction. However, due to uncertainties in the initial density, the scatter in the data is very large. The dilatometry measurements planned in the accelerated aging project should provide data of much greater accuracy to test the models. Robi Mulford will give a detailed account of these density measurements as well as the various ways to analyze the data.

[18] One of the big unknowns in Pu aging is whether this material will also exhibit void swelling. Pu has a damage rate three orders of magnitude lower than generated in the core of fast neutron reactors, in which most of the void swelling experiments had been carried out. So we funded one study at PNNL. to collect swelling data at the lowest dose rates available. Indeed, void swelling does continue to dose rates as low as the one encountered in the accelerated aging program. However, the incubation dose for the onset of void swelling decreases with dose rate, but of course, not with time.

[19] The standard void swelling models, unfortunately, do not produce an incubation stage at all, because they do not simulate the nucleation process realistically. We have started a task to develop a void nucleation code, and we have now demonstrated that this code does generate the dose dependence of void swelling, with an incubation period, a transition stage, and finally the 


\section{UNCLASSIFIED}

steady-state swelling regime. We find that the dislocation density has a dramatic effect on the length of this incubation dose as well as the evolution of the void size distribution. This agrees with the experimental swelling data for cold-worked and solution annealed stainless steels.

[20] The evolution of the void size distribution also changes dramatically from low to high dislocation density. The dislocation density is known to change with dose, and we are presently combining models of dislocation evolution with void nucleation.

[21] Another aspect of void nucleation is that small voids should become unstable and collapse into dislocation loops and stacking fault tetrahedra. But how does this conversion take place? Is there an activation barrier? To find answers to these questions, Brian Wirth has begun to perform MD simulations of vacancy clusters. An example is shown for Al. Interestingly, instead of seeing the conversion process, he finds that the cluster breaks up into smaller ones. Instead of emitting one vacancy at a time, a void may dissociate into smaller clusters. Well, there are always surprises.

[22] In conclusion, I want to emphasize again that we have made advances on many fronts. We have achieved in this program a very close coupling between basic experiments, analysis of surveillance data from stockpile materials, advancing theory at the most fundamental level, and modeling of the microstructural evolution that forms the basis for predicting property changes. 


\section{Modeling of Plutonium Properties and Aging}

\section{Annual Program Review}

March 19-23, 2001

\section{W. G. Wolfer}

With contributions from

Maria Caturla, Allison Kubota, Andrew Quong, Babak Sadigh, Phil Sterne, Mike Surh, Charlene Schaldach, \& Brian Wirth 


\section{Three Modeling Areas: \\ Radiation Effects, Phase Stability, Data Analysis}

Underline are changes made during last year

- Radiation Effects Tasks

Primary Defect Production

Defect Cluster Formation

Void Swelling

Helium Effects
Caturla, $\underline{\text { Kubota }}$

Caturla, Wirth, Wolfer

Surh, Schaldach, Wolfer

Wolfer, Surh

- Phase Stability Tasks

Thermochemistry of Pu Alloys Wolfer

Thermophysical Properties of Pu and its Alloys Quong, Sadigh

Soft Phonon Modes and Martensitic Transformation Quong, Sadigh

Kinetics of Phase Transformations Wolfer, $\underline{\text { Bulatov }}$

- Analysis and Interpretation of Experiments

Positron Annihilation Spectroscopy

Analysis of Void Swelling Data

Photon-electron Spectroscopy and XAFS

Isochronal Annealing Simulations
Sterne, Wirth

Wolfer, Garner (PNNL)

Quong, Surh, Tobin

Wirth 
UNCLASSIFIED

\section{Different age-related contributions \\ to density changes have been considered and analyzed}

Experimental evidence exists for the initial lattice parameter change, and for helium bubble swelling.

So far, no evidence for void swelling or phase transformation.

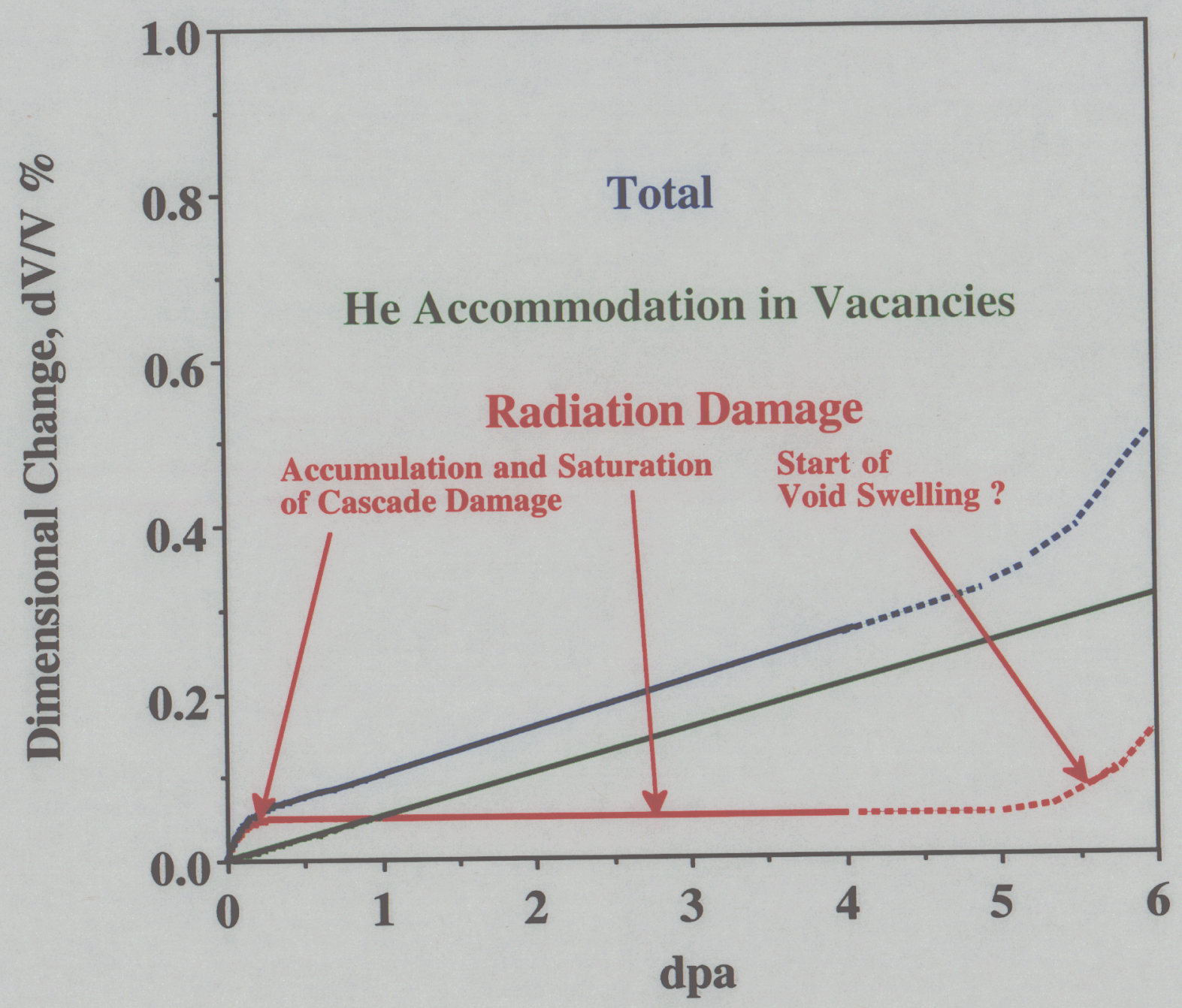




\section{Last year, we performed the first 10 Million Atom MD Simulation} of a $80 \mathrm{keV}$ Collision Cascade in $\mathrm{Pb}$

Cascade break up into three sub-cascades in this particular case. To acquire statistical data requires many more such simulations.

Collisional Stage at 0.33 ps

Atoms with Kinetic Energy $>1 \mathrm{eV}$ are shown
Cooling Stage at 183 ps Atoms with Kinetic Energy $>0.05 \mathrm{eV}$

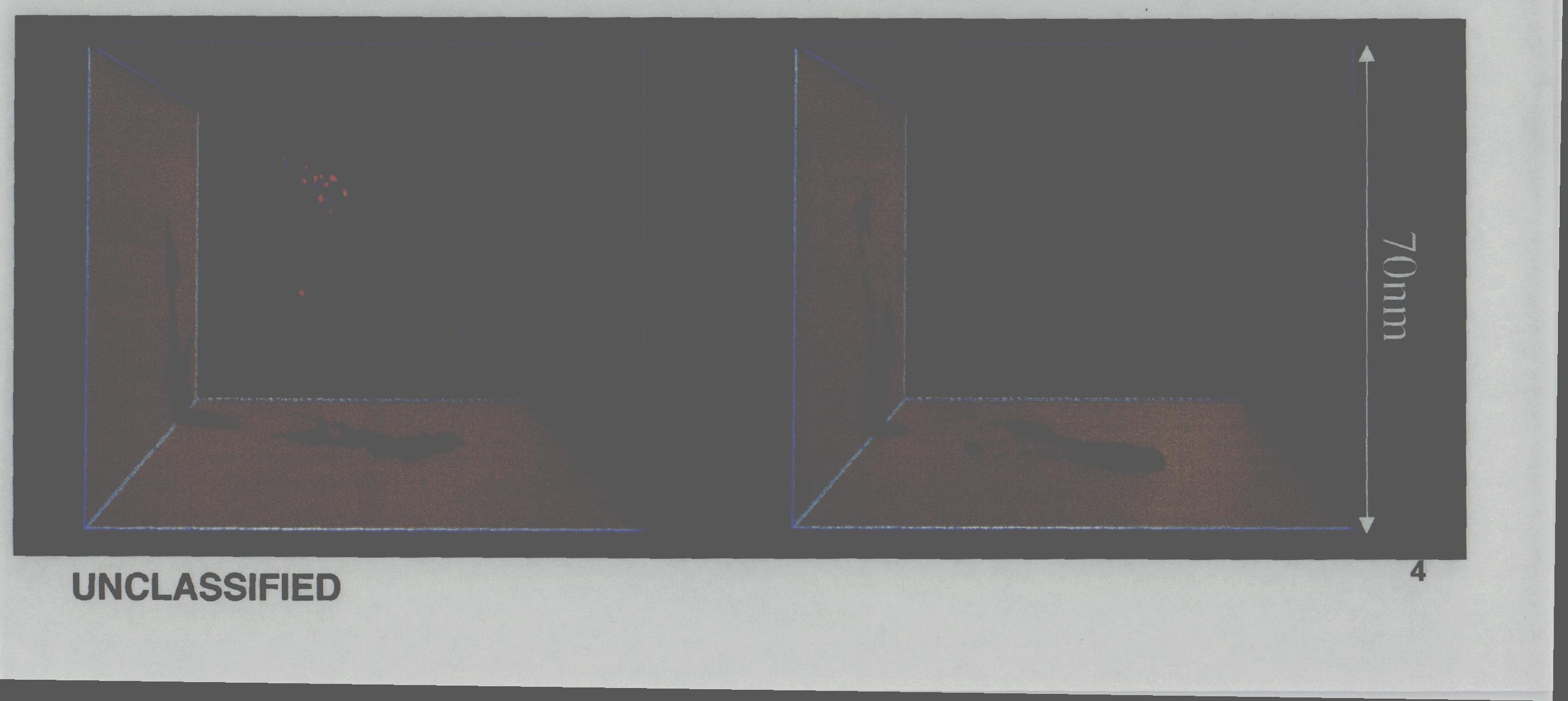


UNCLASSIFIED

\section{The new Baskes MEAM potential for Pu has been implemented in Radiation damage codes}

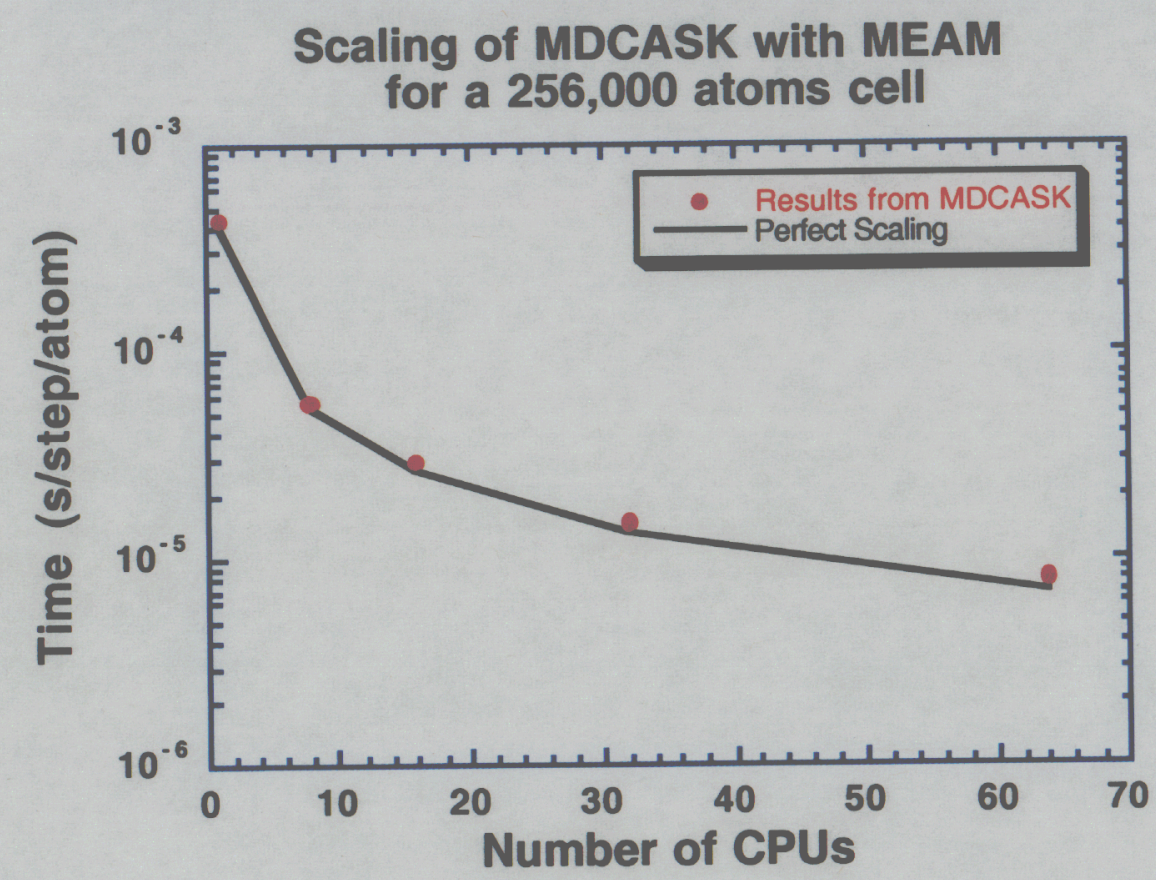

Predicted displacement Energies are somewhat lower than expected.

\section{Allison Kubota \& Maria Caturla}

Excellent scaling has been obtained with the modified MD code.

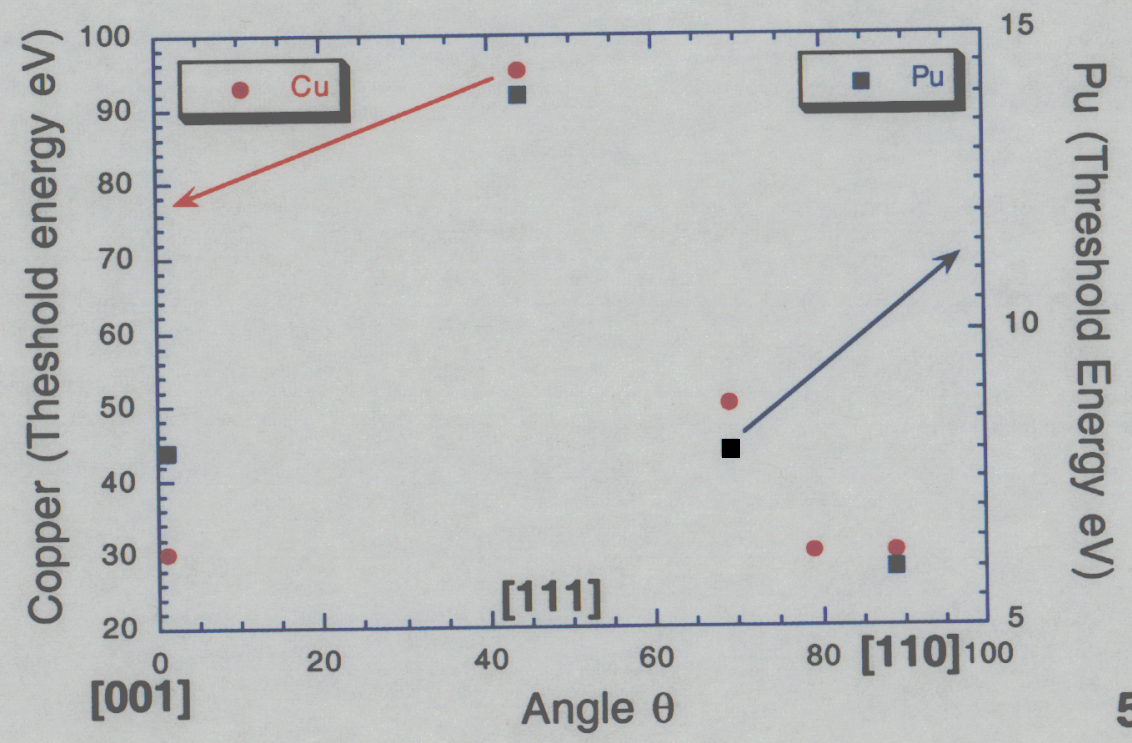

UNCLASSIFIED 

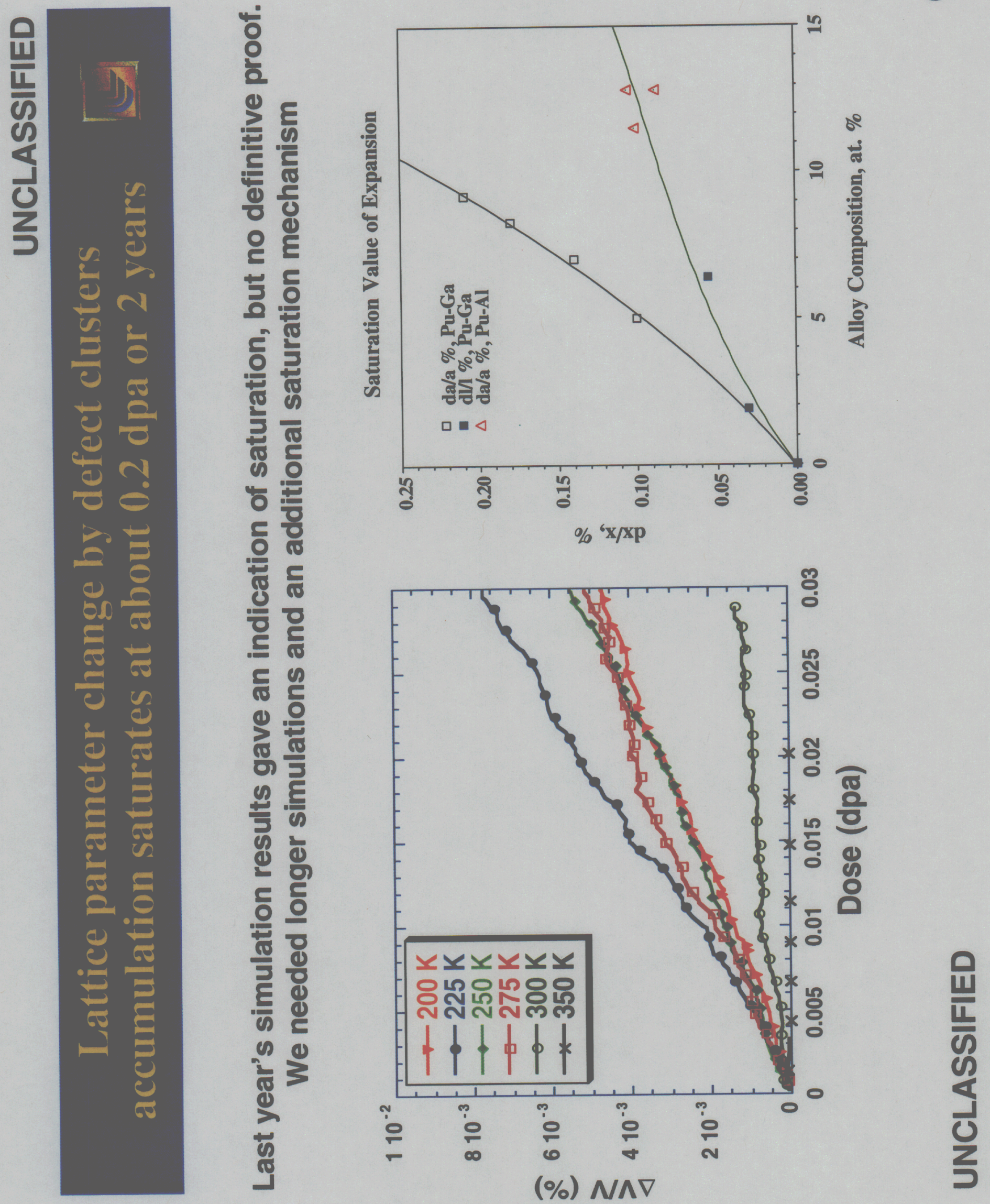


\section{UNCLASSIFIED}

\section{Cascade overlap model has been implemented for kMC simulations, and saturation is now obtained}

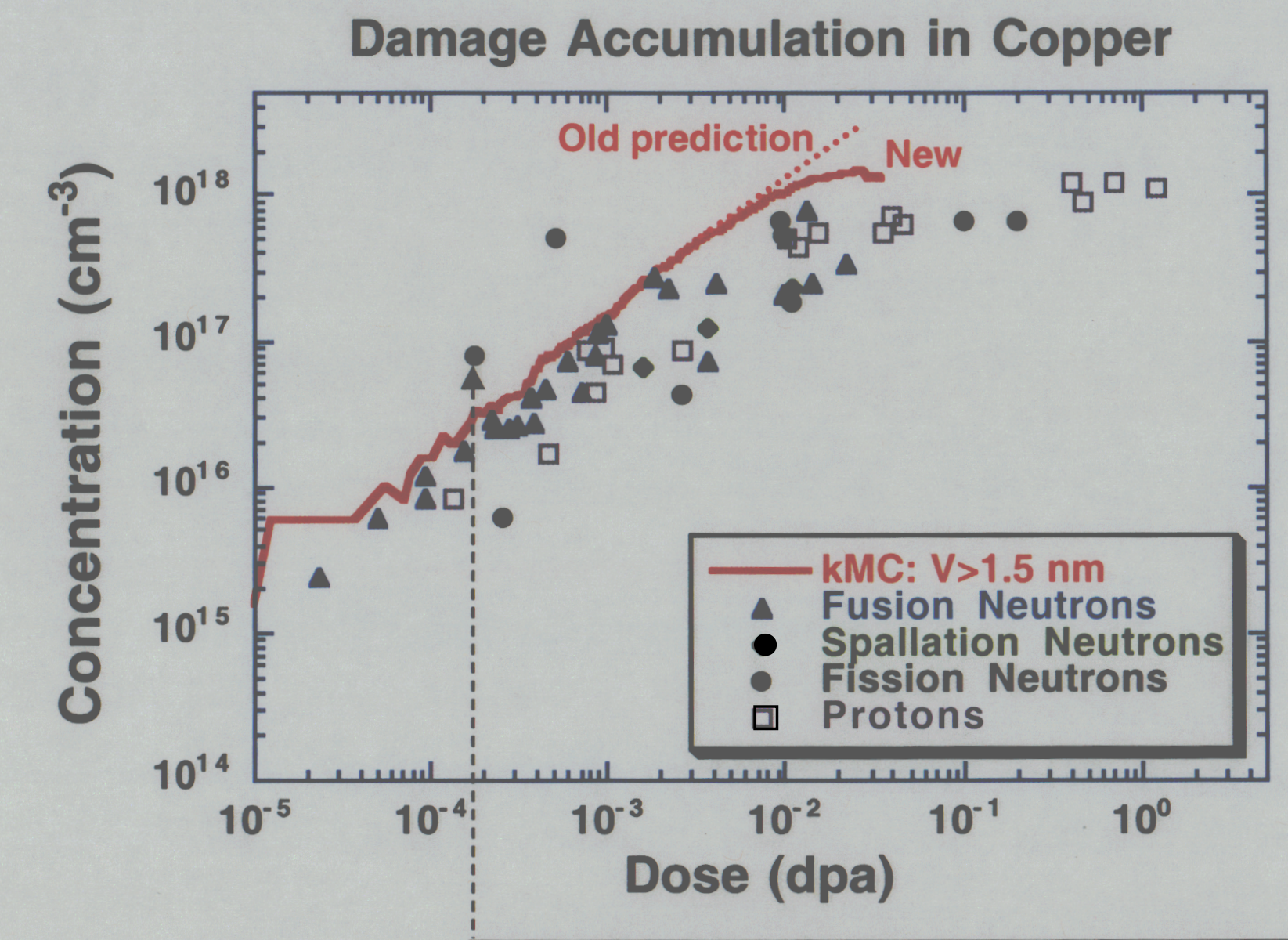

$95 \%$ of the observed clusters are SFT with a side length of $>1.5 \mathrm{~nm}$. Saturation is found at about 0.1 dpa. However, the observed $\Delta \mathrm{a} / \mathrm{a}$ in $\mathrm{Pu}$ is $\mathbf{2 5}$ times larger than predicted. 
UNCLASSIFIED

\section{Significant progress has been made on electronic structure methods for $\mathrm{Pu}$}

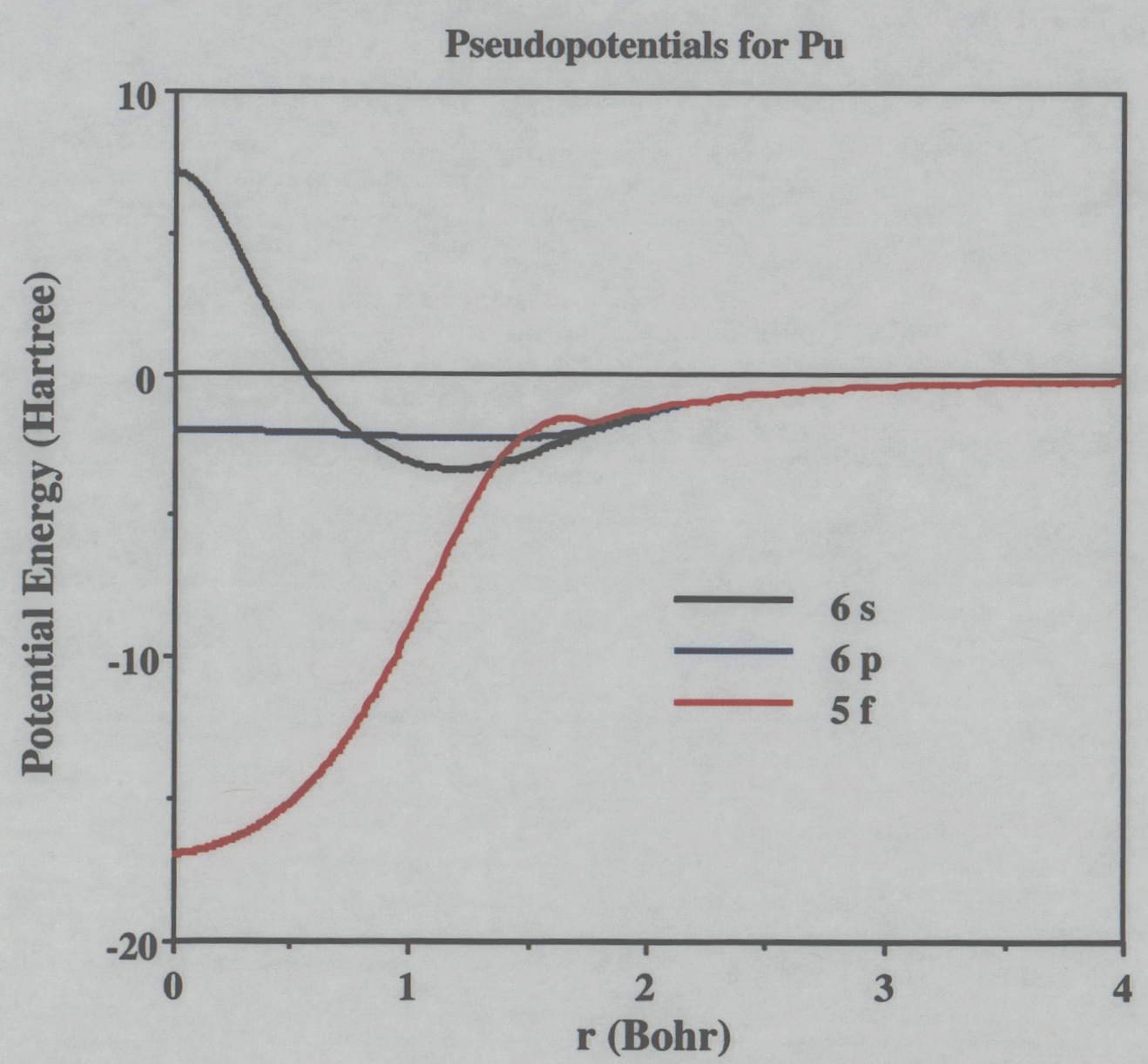

UNCLASSIFIED
Objectives:

Develop an electronic structure method for actinides.

Make it efficient so it can be applied to hundreds of atoms. Couple it to MD methods. Progress:

Spin-polarization has been implemented and codes parallelized.

First good pseudopoentials for Pu (speed-up tenfold). Plan:

Refine pseudopotentials. Include relativistic corrections and spin-orbit coupling. Develop empirical potentials

Babak Sadigh 
UNCLASSIFIED

\section{Spin-polarization effects seem to be the key to explain the low density of the delta phase}

Soderlind demonstrated that spin-polarization renders $\delta$-Pu antiferromagnetic. Large magn. moments for Pu atoms are predicted in $\delta$.

\begin{tabular}{l|l|l}
\hline Method & $\begin{array}{l}\text { Bulk } \\
\text { Modulus } \\
(\text { Gpa })\end{array}$ & $\begin{array}{l}\text { Lattice } \\
\text { Parameter } \\
\text { (nm) }\end{array}$ \\
\hline LMTO & $\mathbf{3 4 . 2}$ & .480 \\
LSDA & $\mathbf{3 1 . 1}$ & .452 \\
GGA & $\mathbf{2 6 . 2}$ & .489 \\
Exp. & $\mathbf{3 0}$ & .464 \\
\hline
\end{tabular}

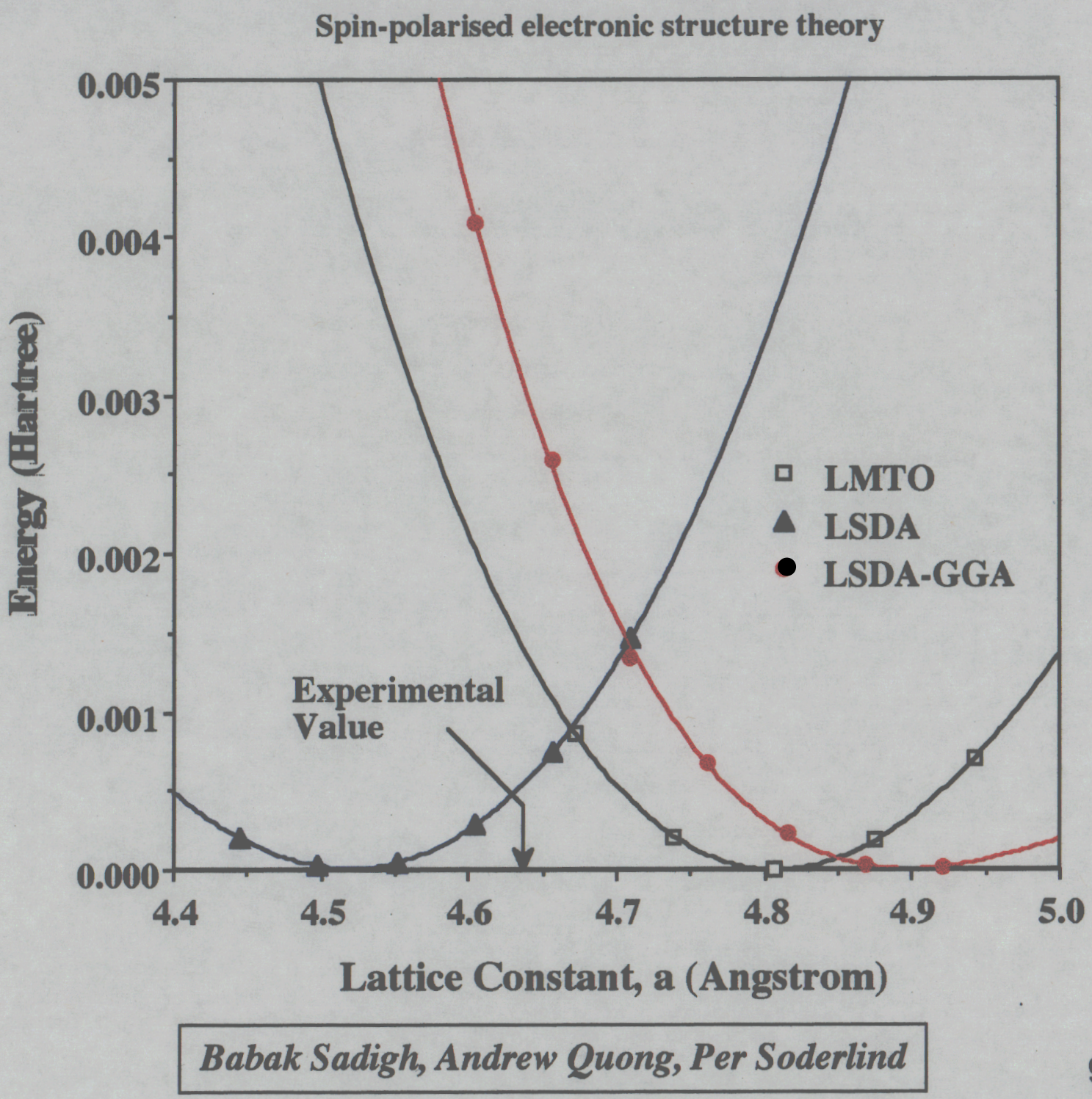

UNCLASSIFIED 


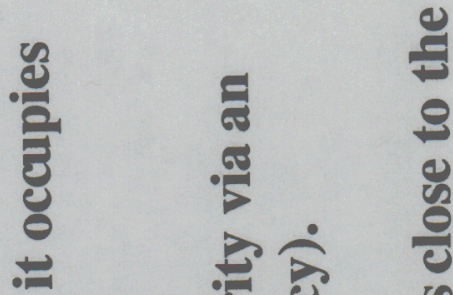

$\because \quad \stackrel{9}{-1}$

है

$\rightarrow \quad$ อ

2

-

.5

- क

i

$\frac{1}{2} \frac{8}{6}$

늘

है II

$\frac{\pi}{\tau}$
욜

.

ㄹ.

.

晋

है



สิ

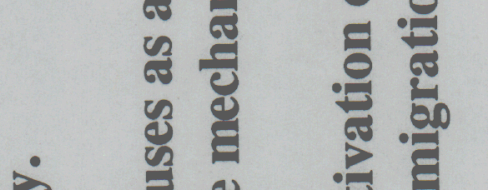

¿

를

흘

$\hat{i} \underset{F}{\circ}$

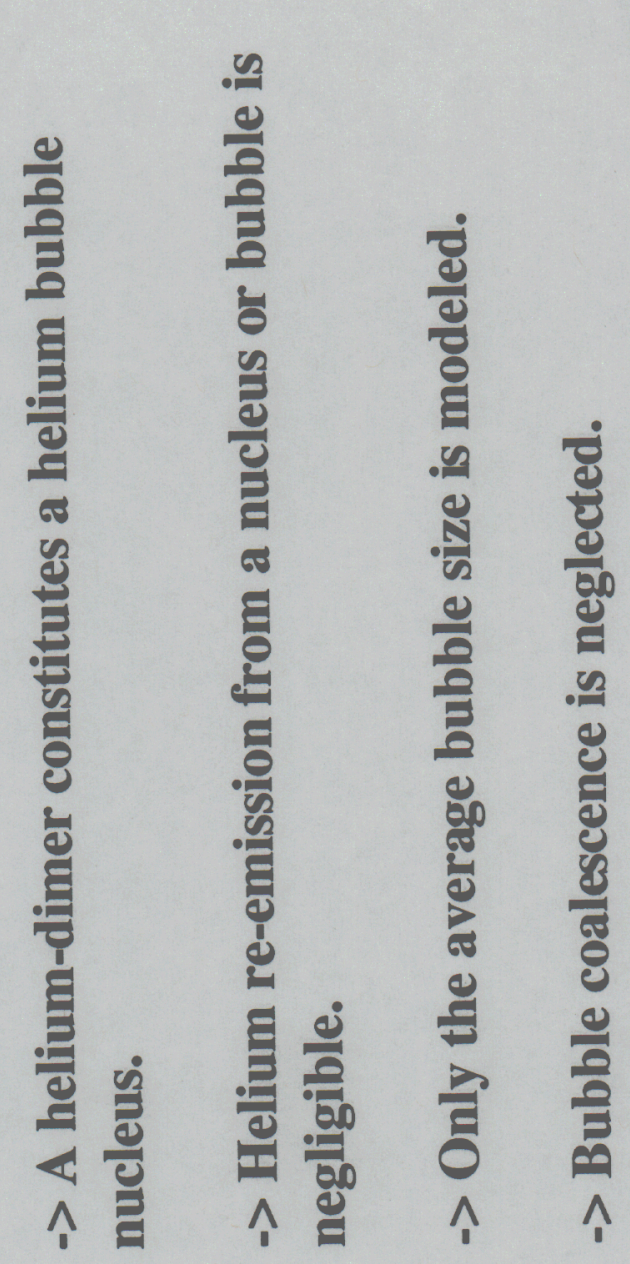

를

울

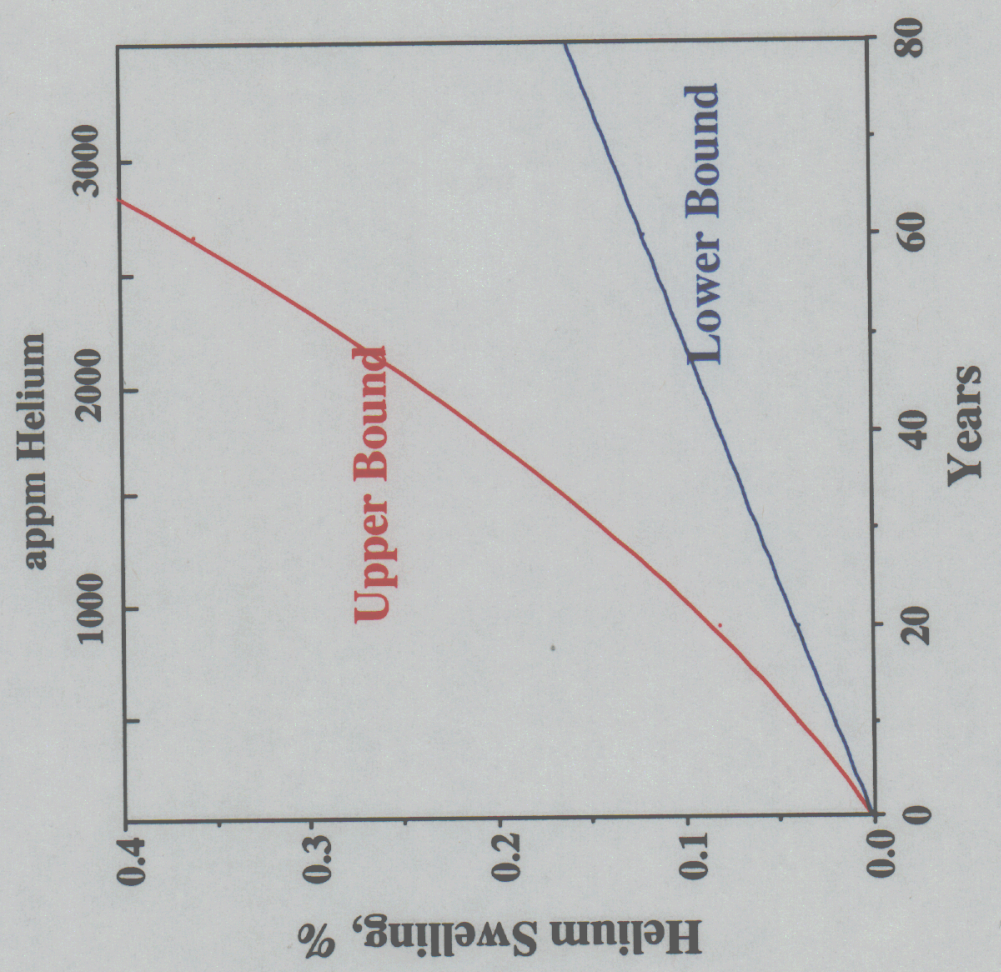

$\frac{0}{4}$ 


\section{UNCLASSIFIED}

\section{The initial model is supported by the TEM observations}

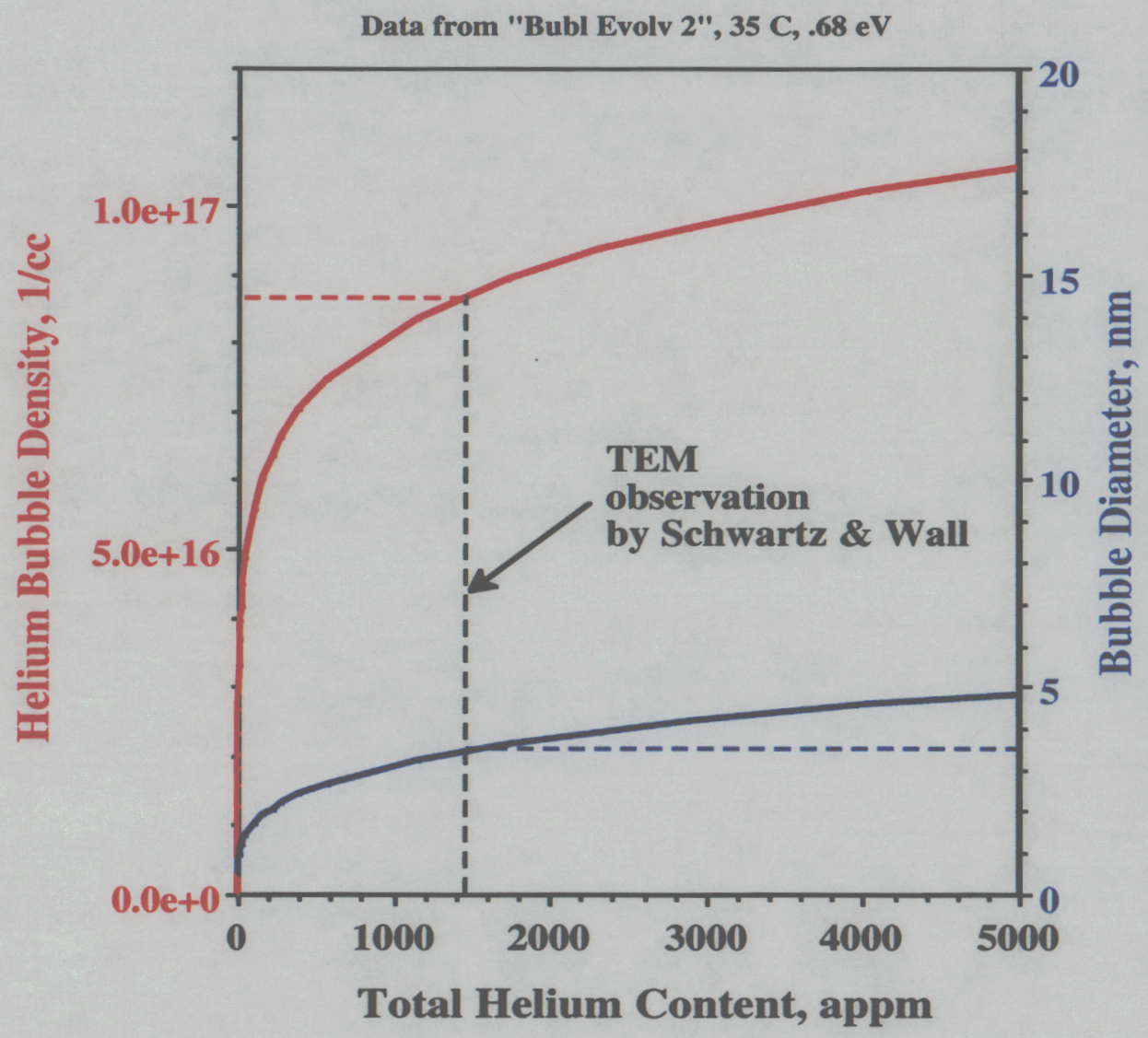

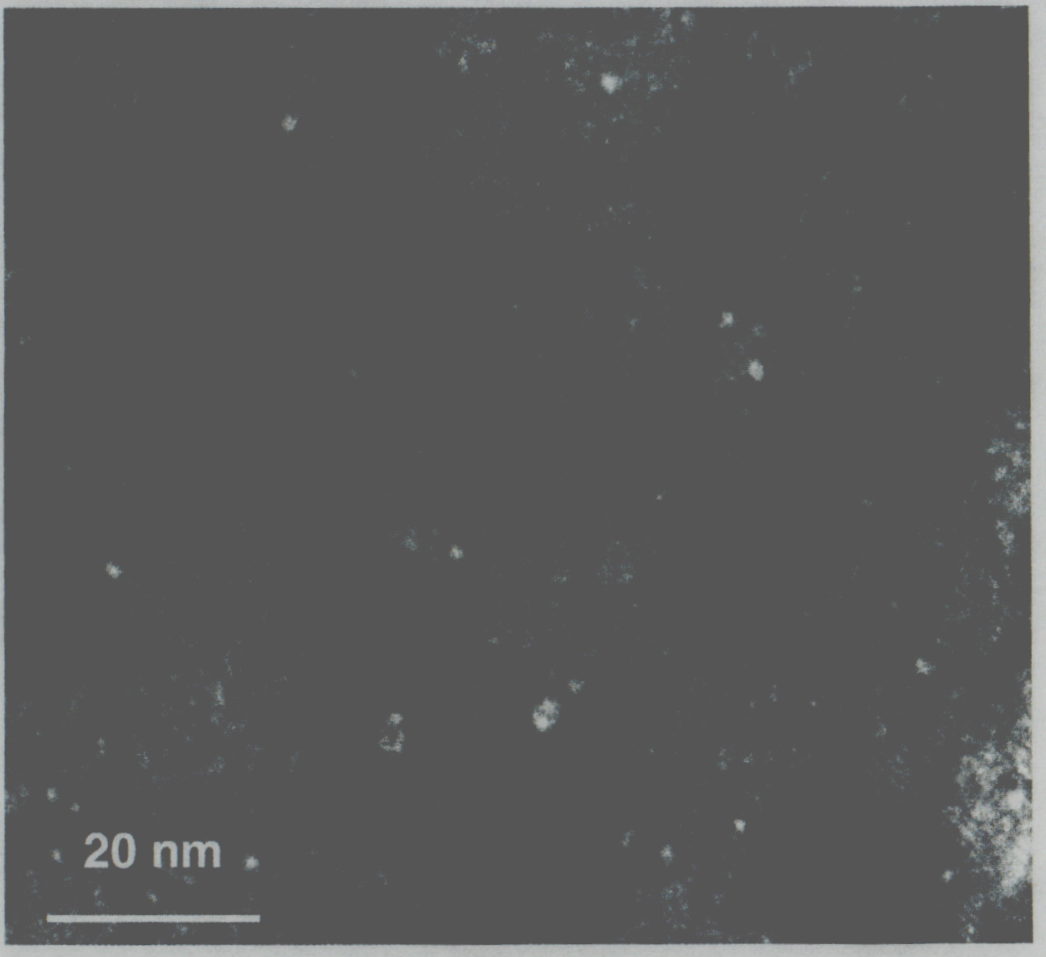

Mark Wall and Adam Schwartz 

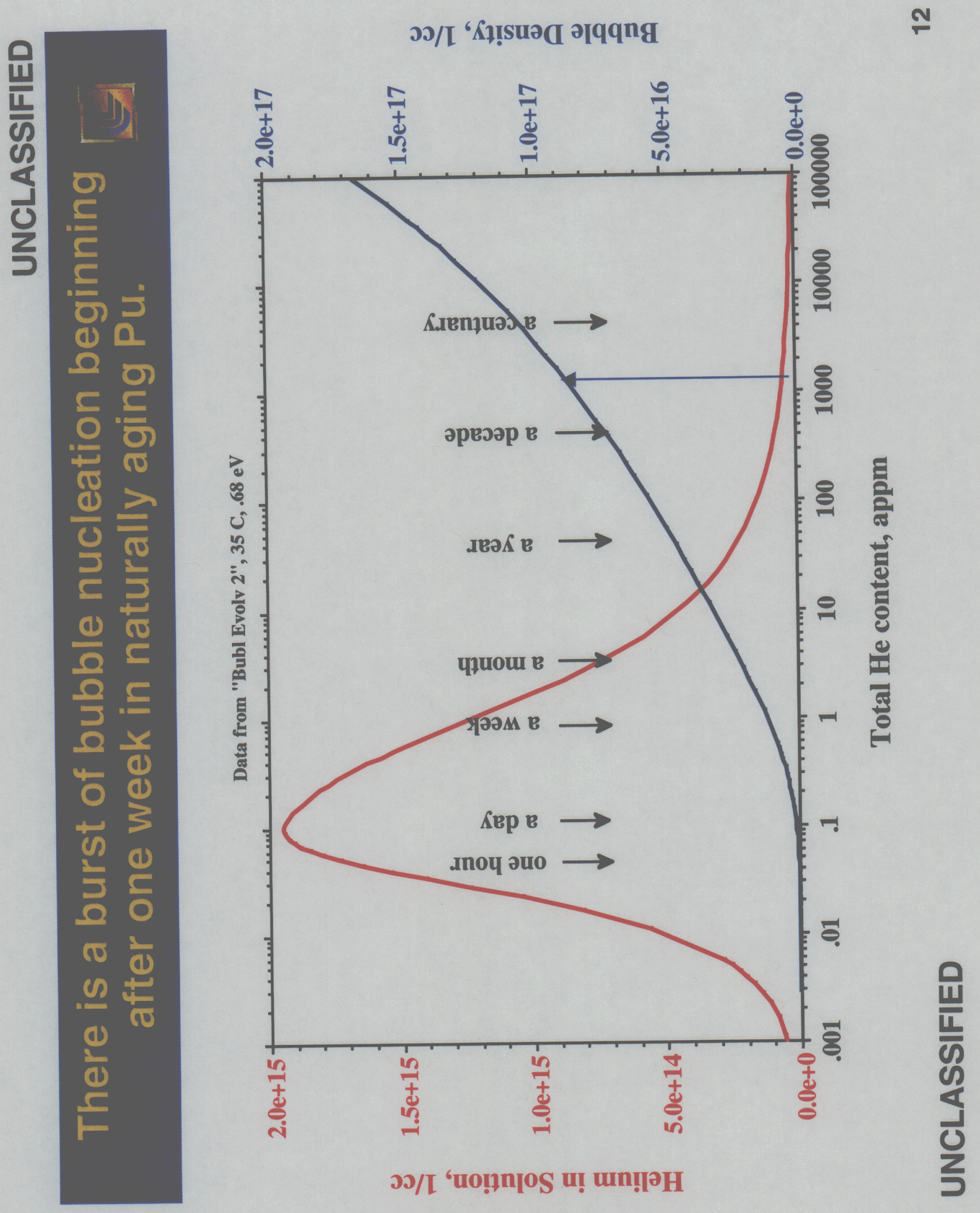


\section{UNCLASSIFIED}

\section{The "final" bubble density depends on storage temperature and enrichment.}

To compensate for accelerated aging, the storage temperature should be higher by about $40 \mathrm{C}$ to obtain the same bubble density. This "temperature shift" is twice as large as for void swelling.

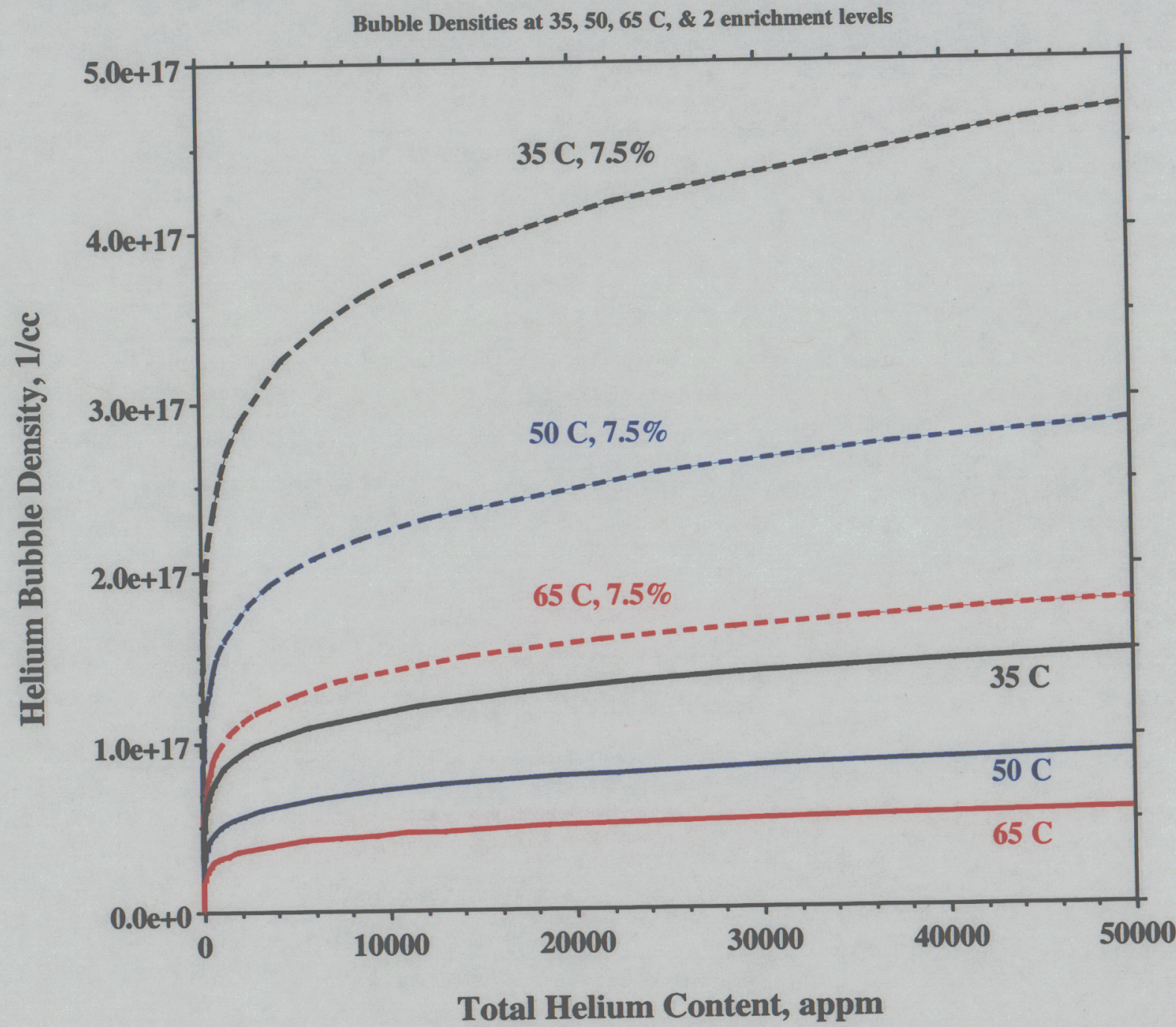

UNCLASSIFIED 
UNCLASSIFIED

\section{High Pressures and Helium Densities exist in Bubbles of nm Size}

Equilibrium bubbles are defined by

$2 \gamma / \mathbf{R}=\mathbf{p}$.

The gas pressure is non-ideal.
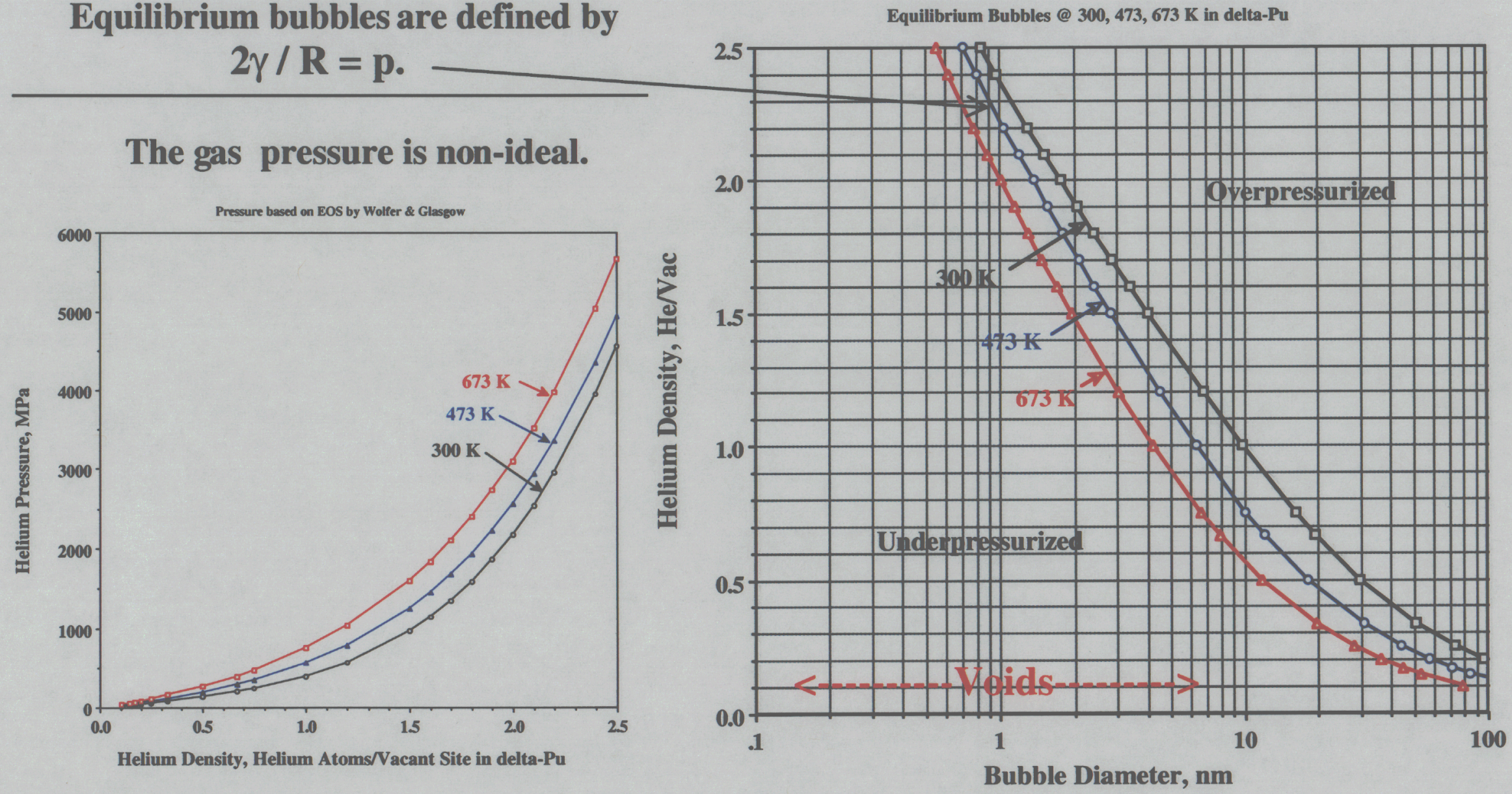
UNCLASSIFIED

\section{Annealing of equilibrium bubbles without coalescence produces only small changes}

PAS studies on annealed Pu indicate a small decline of helium pressure with temperature.

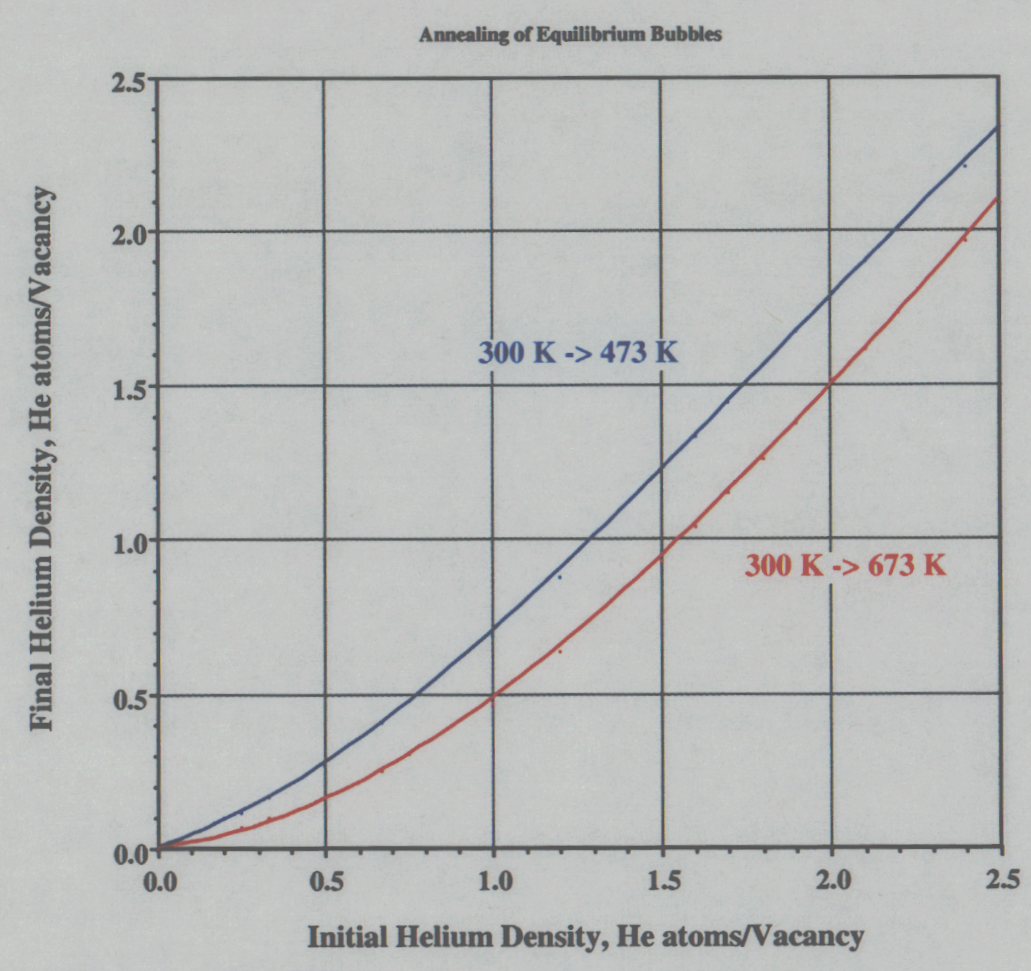

The increase in diameter, however, is too small to be easily observable.

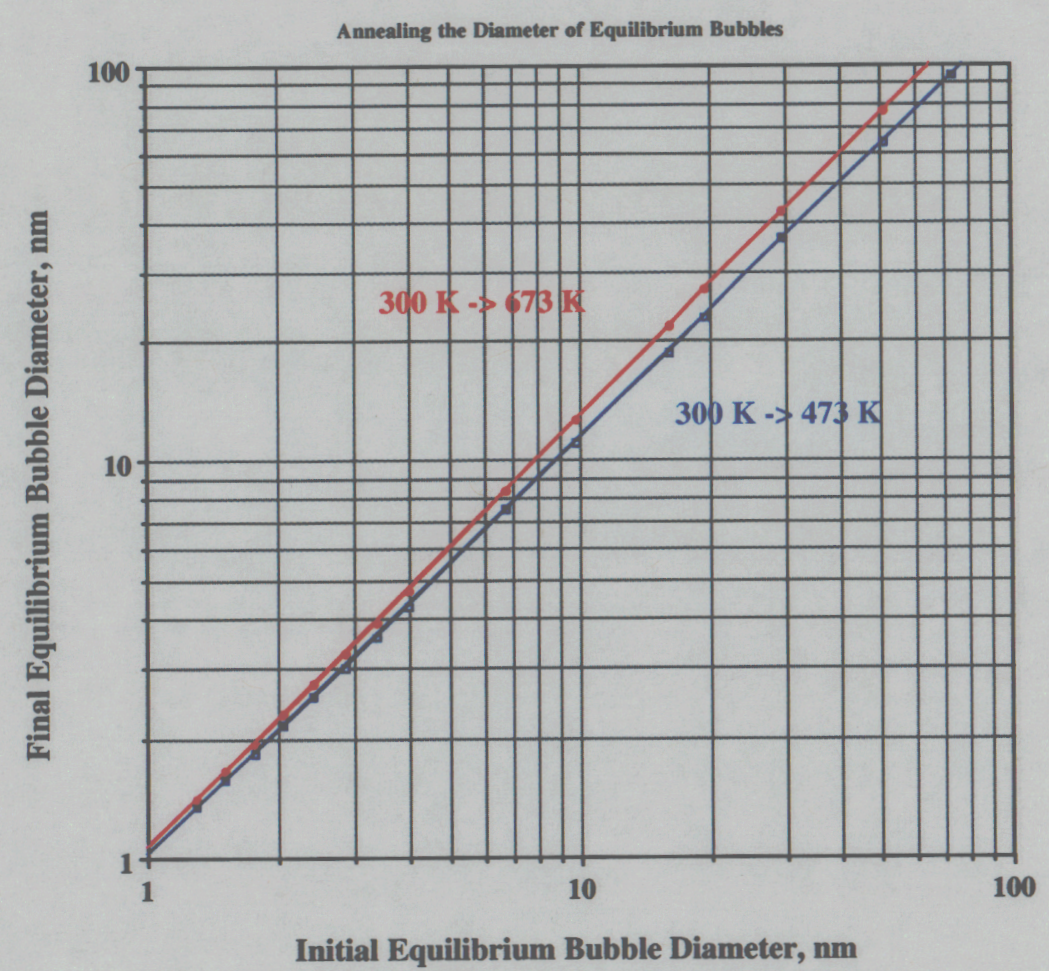


UNCLASSIFIED

\section{The bubble density can be used to estimate the helium diffusion coefficient.}

The lower activation energy, $0.54 \mathrm{eV}$, is close to the Stage III value obtained from isochronal annealing experiments, and from high-temperature bubble swelling studies at AWE.

The higher value is a theoretical estimate by Wolfer.

What is Stage III ?

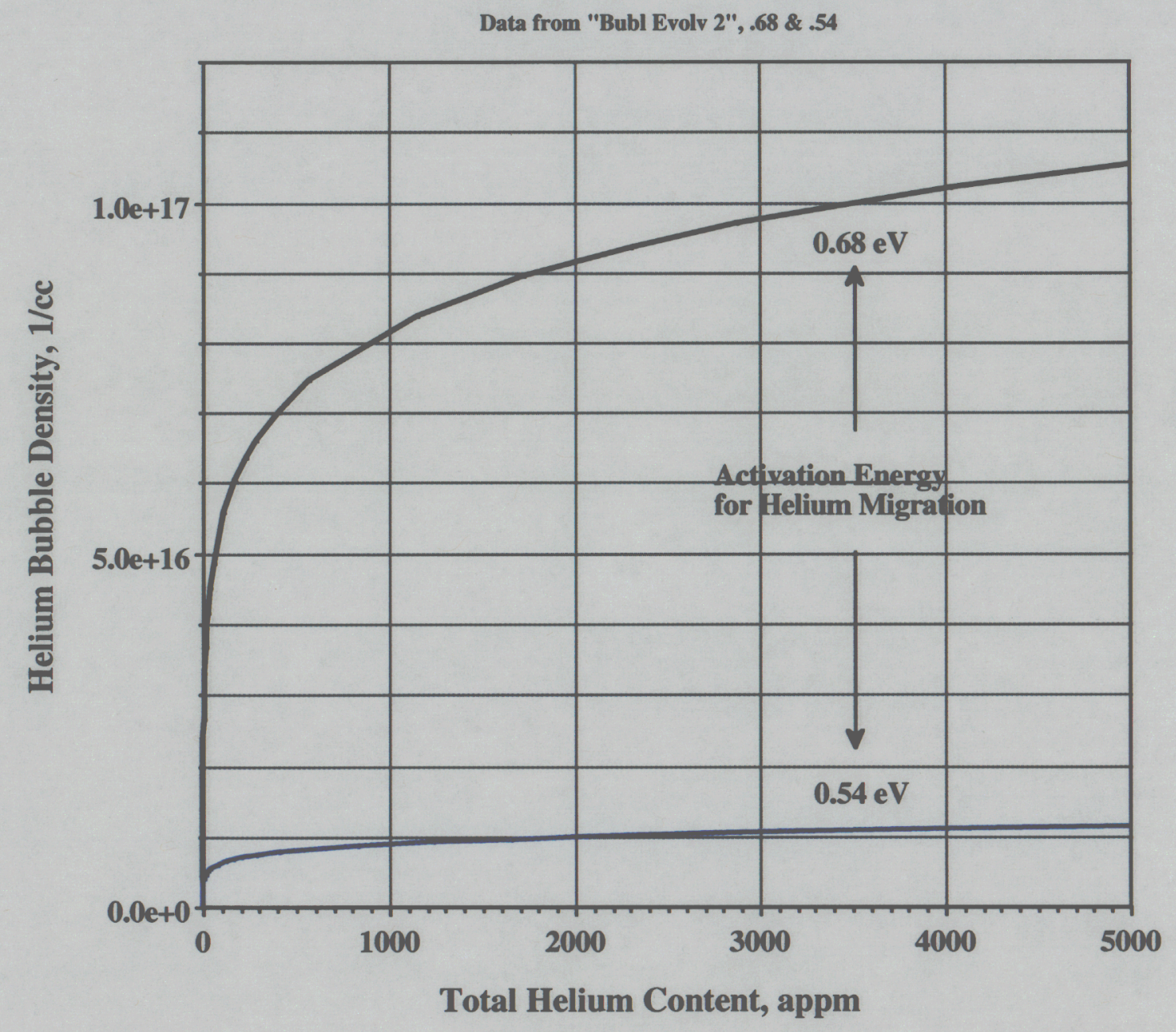

UNCLASSIFIED 
UNCLASSIFIED

\section{Helium Bubble Swelling is predicted to be nearly linear with age, and very small}

The surveillance

data are not compelling evidence For bubble swelling, but at the same time, not inconsistent with model predictions, TEM results, and PAS analysis.

Accelerated aging experiments will provide high precision density changes.

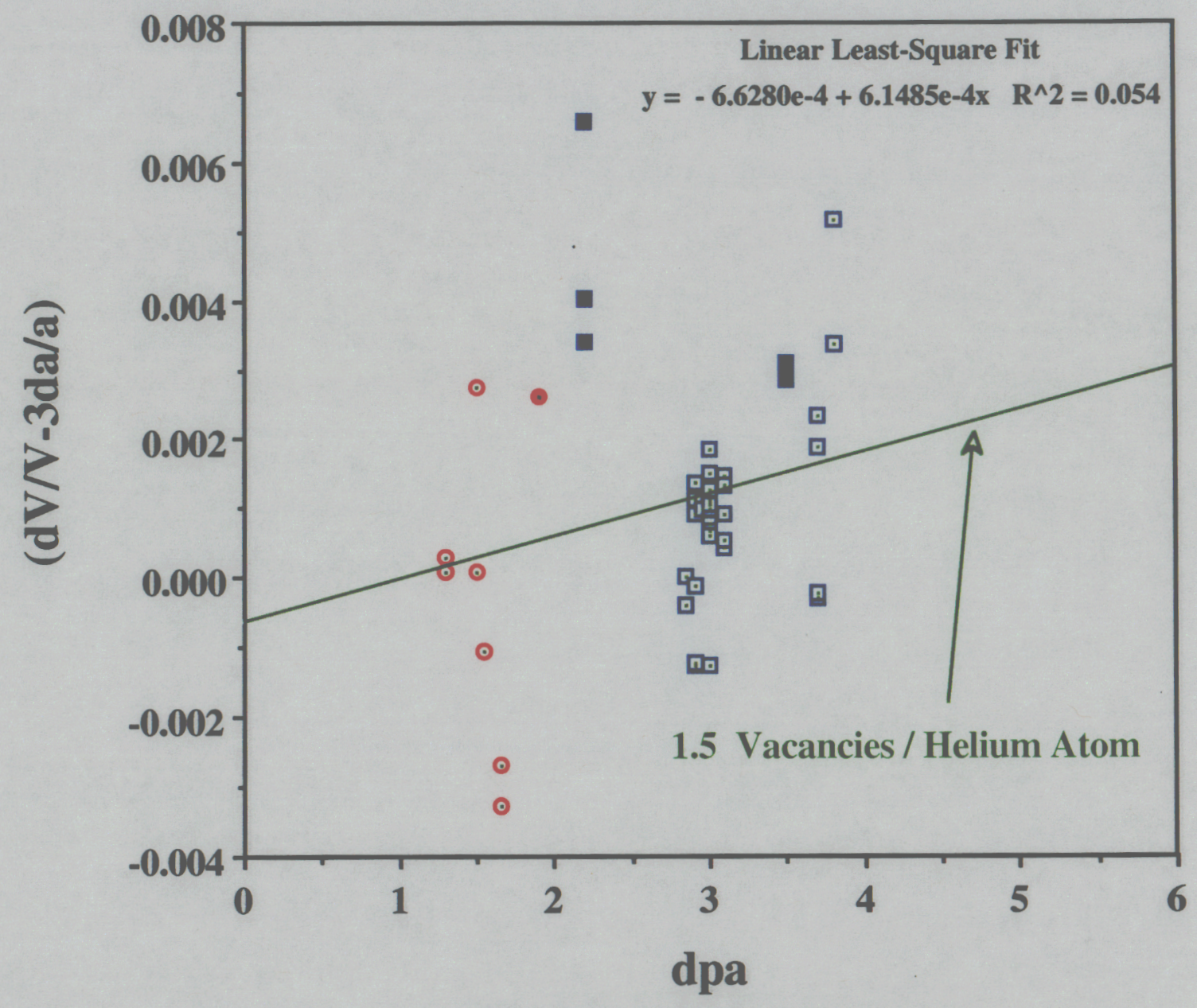

UNCLASSIFIED 


\section{A crucial question for accelerated aging is the onset of void swelling and its extrapolation to lower damage rates $\$$}

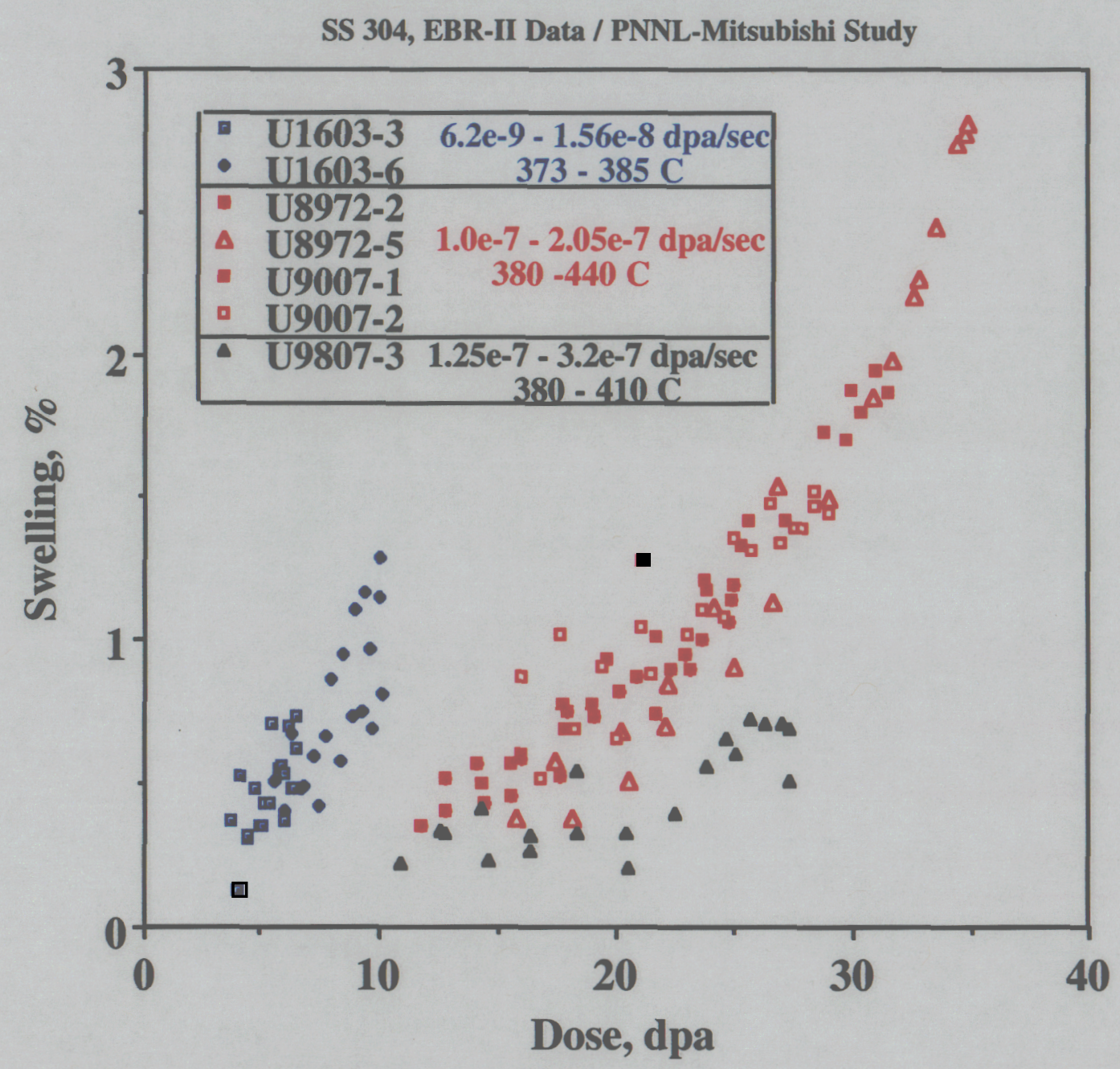

Analysis of reactor data has revealed that void swelling occurs at dose rates as low as in $\mathbf{P u}$, but it begins at lower doses the lower the dose rate. However, previous void swelling models do not treat incubation. 


\section{UNCLASSIFIED}

\section{New simulation results show incubation, and also}

confirm the strong effect of initial dislocation density.
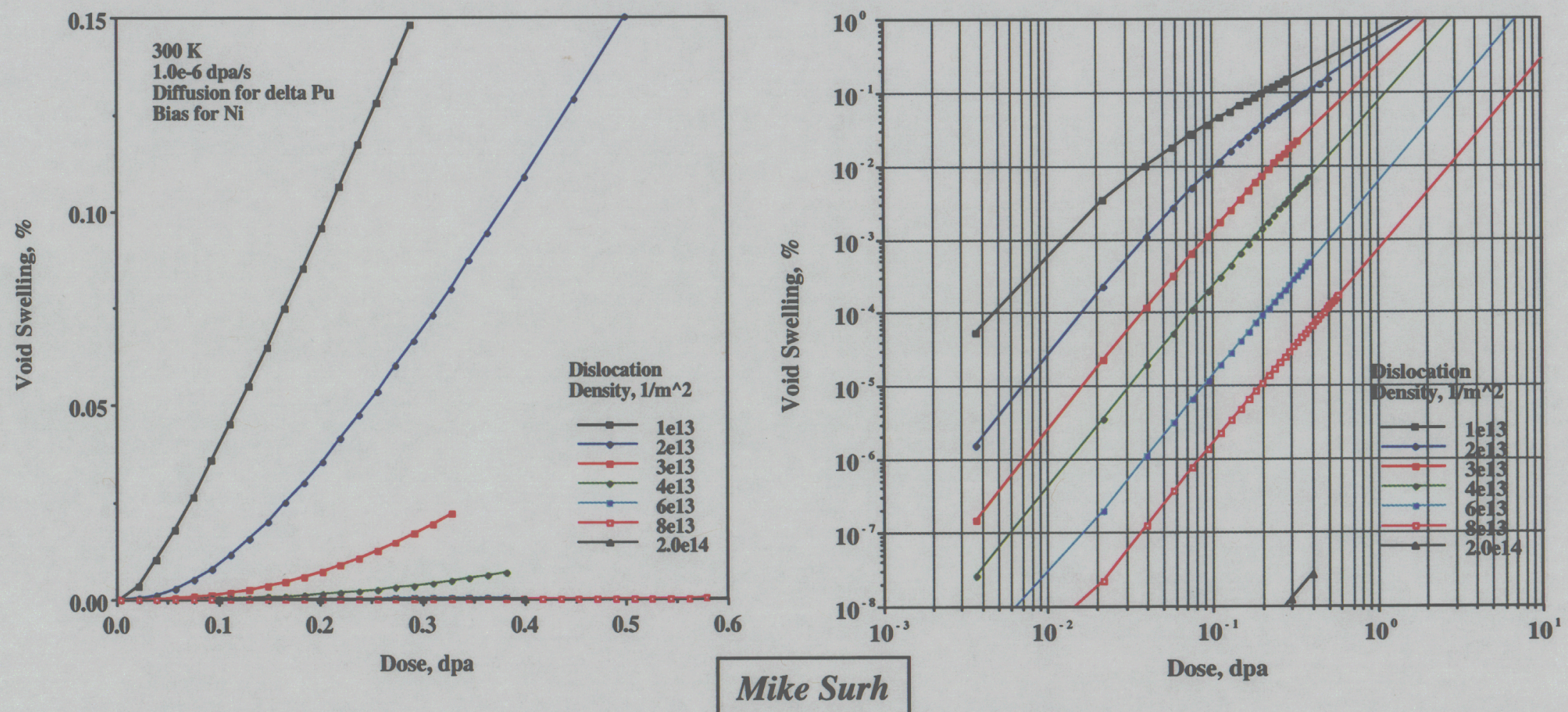

The code predicts for the first time a realistic incubation stage for void swelling followed by a linear stage. 
UNCLASSIFIED

\section{A dramatic change of void nucleation occurs at higher dislocation densities}

Data from "Surh 1 D"

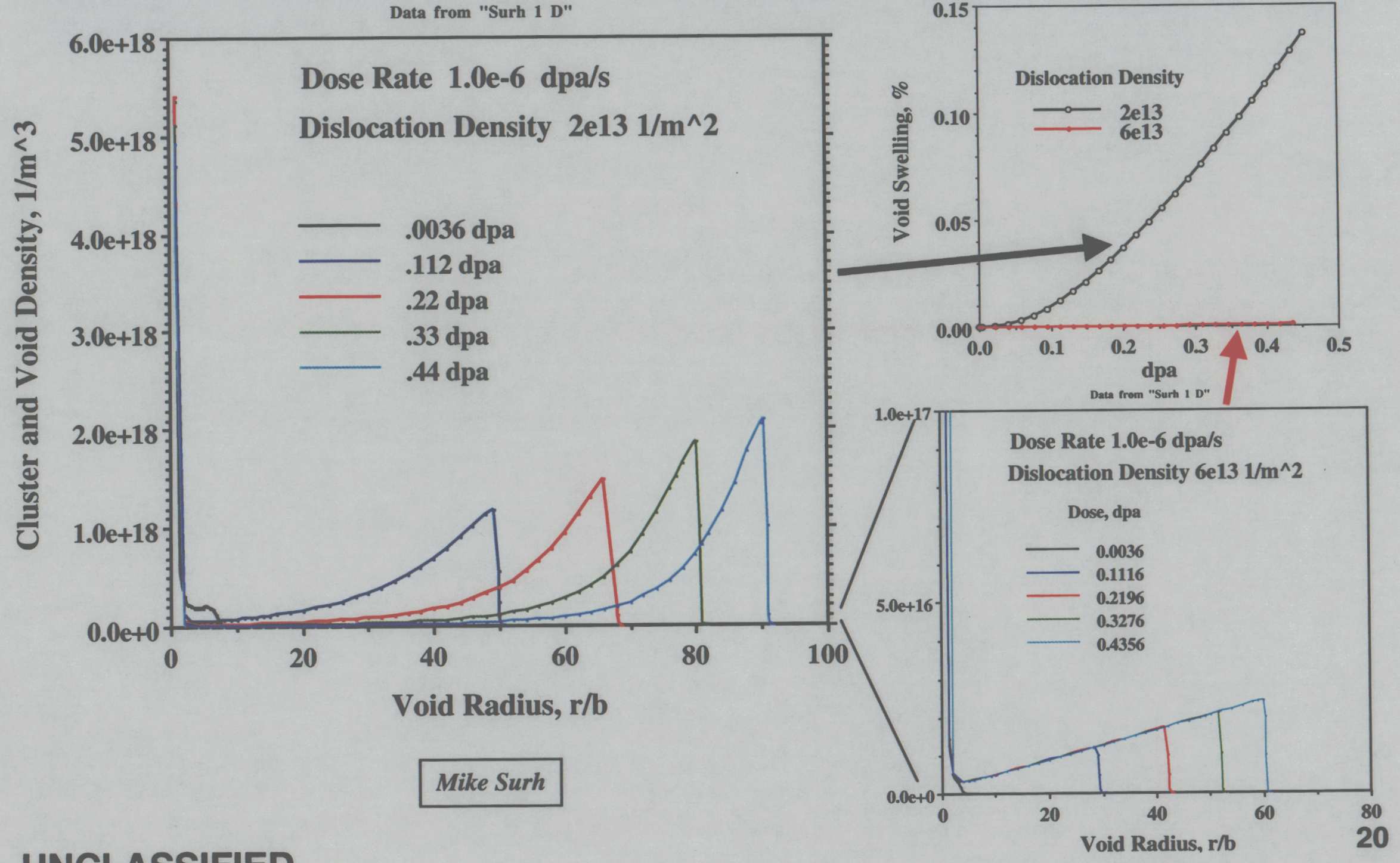

UNCLASSIFIED 

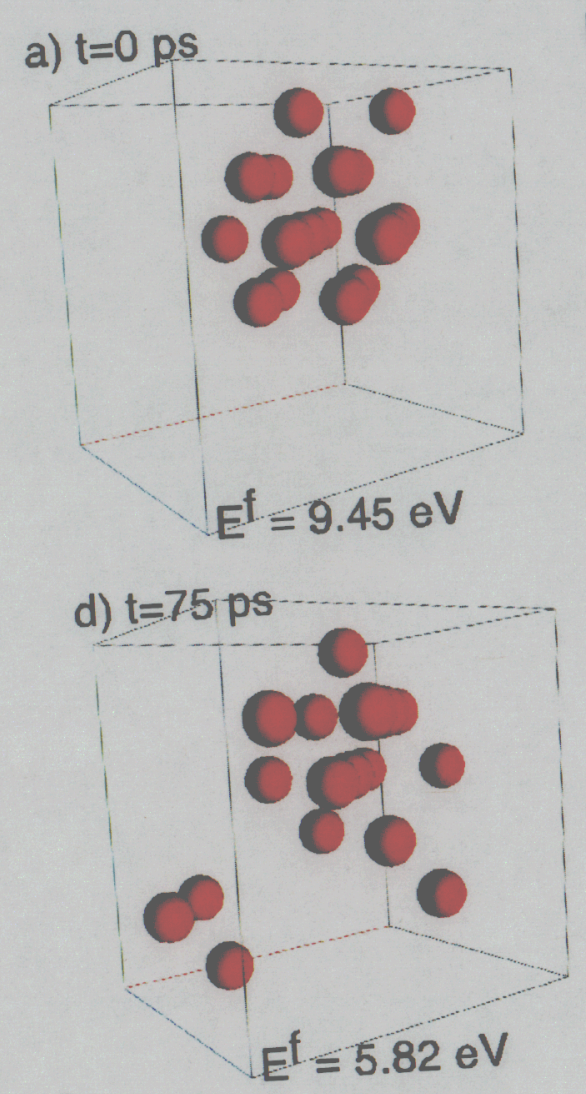

b) $t=25$ ps

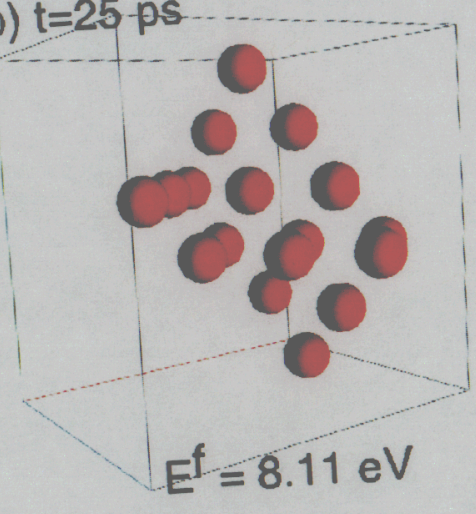

e) $t=100-p s$

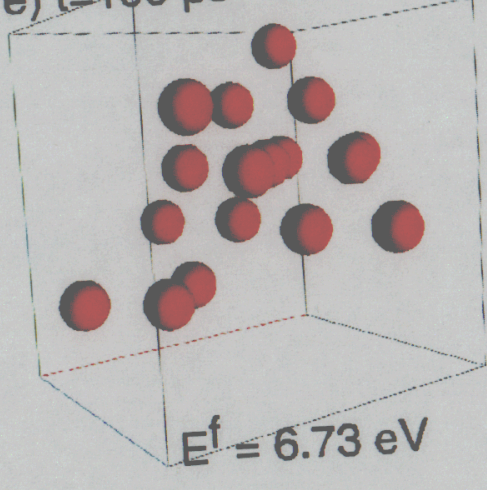

c) $\mathrm{t}=50$-ps
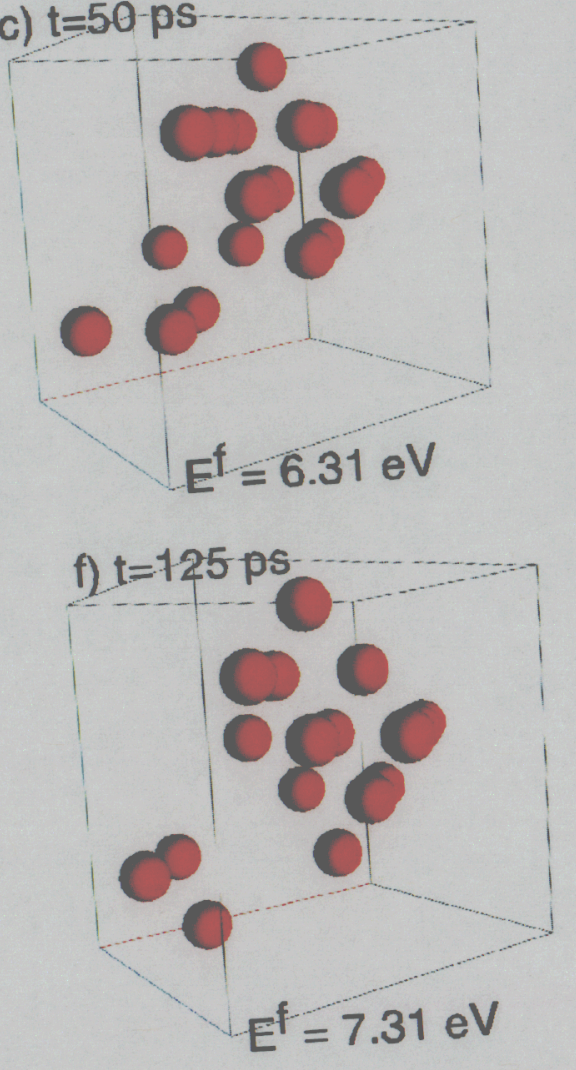

MD simulation of a 17-vacancy cluster in $\mathrm{Al}$ at $600 \mathrm{~K}$.

The cluster sheds a tri-vacancy, instead of one vacancy at a time as previously assumed. Such reaction pathways will reduce void nucleation. Similar calculations are planned for $\mathrm{Pu}$.

Brian Wirth 


\section{Summary of Accomplishments}

- The computational studies are strongly linked to the experimental efforts in Pit surveillance and in accelerated aging.

- Major advances have been made in the underlying science of Pu.

- Electronic structure theory for Pu has experienced two major breakthroughs: Spin-polarization effects explain $\delta$-Pu, and use of pseudo-potentials will enable determination of defect properties.

- Cascade simulations can now be done on Pu with the MEAM.

- TEM, PAS, and Immersion Density measurements on aged stockpile material are tracking helium bubble formation and guide the modeling of helium-induced property changes.

- Our initial model for helium bubble nucleation and growth is remarkable successful in explaining the observations.

- Incubation of void swelling has been modeled successfully, and shown to be strongly dependent on dislocation density. 